\title{
The risk factors for calcification vary among the different sections of the lower extremity artery in patients with symptomatic peripheral arterial disease
}

\author{
Hankun Yan, Zhihui Chang and Zhaoyu Liu* (D)
}

\begin{abstract}
Background: Peripheral arterial disease (PAD) is associated with considerable mortality and morbidity worldwide. The present study explored the risk factors for arterial calcification among the different sections of the lower extremity in patients with PAD and analyzed their correlations with the extent of arterial stenosis at the corresponding section.

Methods: This study enrolled symptomatic PAD patients from our hospital from March 2017 to March 2018. The lower extremity arterial calcification score (LEACS) and lower extremity arterial stenosis index (LEASI), representing the extent of arterial stenosis, were measured on computed tomography $(C T)$ and the correlations between them were analyzed using Spearman's correlation analysis. The relationships between risk factors and calcification were analyzed among the different sections of the lower extremity artery.

Results: In total, 209 patients were included. The LEACSs of the total lower extremity, aortoiliac artery, and femoropopliteal and infrapopliteal arteries were correlated with the LEASI (all $P<0.05)$, but their correlation was relatively weak in the aortoiliac artery. Univariate analysis showed that hypertension was associated with the total $(P=$ $0.019)$ and aortoiliac $(P=0.012)$ LEACSs. Diabetes was related to both femoropopliteal $(P=0.001)$ and infrapopliteal $(P=$ 0.002) LEACSs. The infrapopliteal LEACS was higher in male patients $(P=0.011)$. After adjustment for age, the above relationships were maintained among the different sections, but not in the total lower extremity artery.

Conclusions: The LEACS is associated with the LEASI in all arterial sections, but that of the aortoiliac artery was relatively weak. Different factors have different effects on calcification among the various sections of the lower extremity artery.

Keywords: Arterial calcification, Peripheral arterial disease, Computed tomography, Lower extremity arterial calcification score, Lower extremity arterial stenosis index
\end{abstract}

\section{Background}

With improvements in living standards, changes in dietary habits, and prolongation of average lifespans, the morbidity rate of peripheral arterial disease (PAD) is increasing every year, which not only gravely affects people's quality

\footnotetext{
* Correspondence: liuzy1226@126.com

Department of Radiology, Shengjing Hospital of China Medical University, NO. 36, Sanhao Street, Heping District, Shenyang City 110004, Liaoning Province, China
}

of life, but also increases their risk of death and limb ischemic events [1-3]. As a characteristic sign of PAD, arterial calcification has also been linked to adverse limb events and poor prognosis of interventional treatment and is associated with increased all-cause mortality [4-6]. Arterial calcification was previously considered a passive degenerative process but is currently recognized to be a complex process actively regulated by various cell molecules [7]. The abnormal deposition of extra osseous

C The Author(s). 2020 Open Access This article is licensed under a Creative Commons Attribution 4.0 International License, which permits use, sharing, adaptation, distribution and reproduction in any medium or format, as long as you give appropriate credit to the original author(s) and the source, provide a link to the Creative Commons licence, and indicate if changes were made. The images or other third party material in this article are included in the article's Creative Commons licence, unless indicated otherwise in a credit line to the material. If material is not included in the article's Creative Commons licence and your intended use is not permitted by statutory regulation or exceeds the permitted use, you will need to obtain permission directly from the copyright holder. To view a copy of this licence, visit http://creativecommons.org/licenses/by/4.0/ The Creative Commons Public Domain Dedication waiver (http://creativecommons.org/publicdomain/zero/1.0/) applies to the data made available in this article, unless otherwise stated in a credit line to the data. 
calcium salt in the human body occurs most frequently in the arterial system and causes arterial calcification $[8,9]$, mainly involving intima calcification and media calcification. However, these two types of arterial calcification cannot be accurately distinguished by conventional computed tomography (CT) [10]. According to the literature, arterial calcification is associated with traditional cardiovascular risk factors, such as diabetes, hypertension, hyperlipidemia, chronic kidney disease (CKD), and aging [11, 12]. Nonetheless, further studies have shown different mechanisms and prognoses of calcification in different vascular beds. Previous work has shown that arterial calcification is related to the apoptosis of vascular smooth muscle cells and macrophages and the release of matrix vesicles [13, 14]. In addition, some studies also showed that different types of arterial calcification (intima versus media) develop through various molecular mechanisms in different vessel types (large elastic versus smaller muscular arteries) and parts (proximal versus distal) $[15,16]$. Allison et al. found that calcification of the thoracic, carotid, and iliac arteries was associated with overall mortality, whereas that of the coronary arteries was associated with cardiovascular disease mortality [17]. However, few studies have focused on the risk factors for calcification in the different sections of the lower limbs.

The relationship between calcification and arterial stenosis in the carotid and coronary vascular bed is well established $[18,19]$. In contrast to calcification in carotid and coronary arteries, arterial calcification of PAD is most prominently located in the media and might even be more pronounced [20]. Therefore, the contribution of lower extremity arterial calcification (LEAC) to arterial stenosis may be different from in the neck and heart, and further exploration is necessary for the relationship between LEAC and the extent of arterial stenosis in patients with PAD. Quantification of LEAC has become readily available through semiautomated protocols using CT scans [21], and it has been widely used to predict mortality and assess the potential for successful interventional therapy [22-24]. In this study, a CT-based calcification score (CS) was used to evaluate the impact of cardiovascular risk factors on calcification in different sections of the lower extremity artery and to determine the correlations between the CS and the extent of arterial stenosis in different sections in patients with symptomatic PAD.

\section{Materials and methods}

This retrospective, observational, single-center study included consecutive patients with symptomatic PAD who underwent CT angiography (CTA) in our hospital between March 2017 and March 2018. Approval for the study was obtained from our Institutional Review Board (IRB approval number: 2018PS225K), and patient consent was waived because of the retrospective nature of the study. We did not include patients presenting with acute embolism or in-stent restenosis or those with a prior amputation above the ankle. Baseline patient characteristics and individual risk factors collected included hypertension, hyperlipidemia, diabetes mellitus, CKD, and history of smoking and drinking.

\section{CT examination}

In this study, CTA was performed with the following scanners: Brilliance iCT 256-slice CT scanner (Philips Healthcare, Cleveland, OH, USA), Ingenuity Core 128slice CT scanner (Philips Healthcare, Cleveland, $\mathrm{OH}$, USA), and Aquilion ONE 640-slice CT scanner (Toshiba Medical Systems, Otawara, Japan). The scan ranged from the abdominal aorta to the plantar artery. Briefly, $100 \mathrm{ml}$ contrast medium (Iohexol, Shuangbei 350; Beilu Pharmaceutical Co., Ltd., Beijing, China) was injected into the middle vein of the right elbow at $4 \mathrm{ml} / \mathrm{s}$ via a 20-gauge intravenous cannula and a dual-cylinder highpressure syringe (Ulrich REF XD 2051; Ulrich Medical $\mathrm{GmbH}$, Ulm, Germany). Then, $40 \mathrm{ml}$ normal saline was infused at the same rate.

\section{Measurement of the calcification score}

The CS was measured from $3 \mathrm{~cm}$ of the abdominal aorta terminal to the ankle joint in plain images. The lower extremity artery was divided into three sections-aortoiliac arterial, femoropopliteal arterial, and infrapopliteal arterial-and each section included left and right corresponding arteries of the lower extremities. The aortoiliac arterial section included the abdominal aorta terminal, bilateral common iliac artery, and bilateral external iliac artery; the femoropopliteal arterial section included the bilateral superficial femoral artery and bilateral popliteal artery; and the infrapopliteal arterial section included the bilateral pretibial artery, bilateral tibiofibular diaphysis, bilateral post-tibial artery, and bilateral fibular artery. LEAC was measured on noncontrast CT scans. Calcification regions with a calcification area $>1 \mathrm{~mm}^{2}$ and density $>130 \mathrm{Hu}$ were identified automatically using the open-source DICOM viewer (v4, OsiriX Imaging Software, Pixeo SARL) of Apple Macintosh (Apple Inc., Cupertino, CA, US). Bone tissues around target arteries from the abdominal aorta to the ankle joint were avoided; the target arterial sections were selected along the abdominal aorta (terminal $3 \mathrm{~cm}$ ), iliac artery, femoropopliteal artery, and infrapopliteal artery. Finally, an overall CS was determined for each patient as the sum of all segmental CSs for both legs. Because the scores were skewed, log-transformed $(C S+1)$ values were used for all statistical analyses. Thus, we defined the $\log _{10}(C S+1)$ values as the lower extremity arterial calcification score (LEACS). Two investigators who were blinded to the clinical status of the patients performed all calcium scoring after a brief training session. 
Evaluation of the inter- and intraobserver variabilities in the determination of the LEACS revealed Spearman correlation coefficients of $0.987(P<0.001)$ and $0.962(P<$ 0.001 ), respectively [25].

\section{Measurement of the extent of arterial stenosis}

In the contrast CT scans of the lower extremity CTA examination, the stenotic arterial sections were assessed independently by two radiologists. On the base of the three above sections, the lower extremity artery of each patient was further divided into 29 segments [26]. The aortoiliac arterial section included the abdominal aorta terminal, bilateral common iliac artery, and bilateral external iliac artery; the femoropopliteal arterial section included the bilateral deep femoral artery, bilateral superficial femoral artery (proximal and distal segments), and bilateral popliteal artery (proximal and distal segments); and the infrapopliteal arterial section included the bilateral pretibial artery (proximal and distal segments), bilateral tibiofibular diaphysis, bilateral posttibial artery (proximal and distal segments), and bilateral fibular artery (proximal and distal segments). According to the lumen stenosis degree, the arterial stenosis degree was classified into nonsignificant stenosis (lumen diameter decreased by $<50 \%$ ) and significant stenosis (lumen diameter decreased by $\geq 50 \%$ ). The lower extremity arterial stenosis index (LEASI) was defined as the total number of arterial segments of the lower extremity with significant stenosis.

\section{Statistical analysis}

Measurement data conforming to the normal distribution are expressed as mean \pm standard deviation, nonnormally distributed data are expressed as median (interquartile range), and count data are expressed as frequency and composition ratio. The correlations of the LEACS and the LEASI in the whole lower extremity and at each section were analyzed using Spearman's correlation coefficient. By taking the median LEACSs (total, aortoiliac, femoropopliteal, and infrapopliteal arteries) as cutoff values, the patients were allocated into high and low calcification groups. Intergroup comparisons were performed using a chi-square test. The risk factors for arterial calcification in the total lower extremity and at each section were assessed by univariate linear regression analysis. Multivariate linear regression analysis was used to determine the independent risk factors associated with the LEACS and obtain the partial regression coefficient (PRC), 95\% confidence interval (CI), and $P$ value. All statistical analyses were performed with SPSS 25.0 software and all statistical tests were two-tailed. $P<$ 0.05 indicated that the difference was statistically significant.

\section{Results}

A total of 209 patients were included in this study. The patient selection process is detailed in Fig. 1. Patients' basic characteristics are shown in Table 1.

According to the lower extremity CTA examination, 627 sections of the lower extremity artery, encompassing a total of 6061 segments, were assessed. Significant stenosis was found in 2639 segments (43.5\%). The total LEACS was associated with the total LEASI $(r=0.459, P<0.001)$. As for each arterial section, the LEACSs of the aortoiliac artery $(r=0.317, P<0.001)$, femoropopliteal artery $(r=0.510, P<$ $0.001)$, and infrapopliteal artery $(r=0.547, P<0.001)$ were all correlated with the LEASI, but their correlation was relatively weak in the aortoiliac artery (Fig. 2).

The correlations among the various arterial sections of the lower extremity (including the aortoiliac artery, femoropopliteal artery, and infrapopliteal artery) and the total LEACS are shown in Table 2. The LEACS at each arterial section of the lower extremity and the LEACS at the left/ right lower extremity artery were strongly associated with the total LEACS. A high correlation was also found in the LEACS between the left and right lower extremities. Among the three sections of the lower extremity, the highest correlation with total LEACS was observed at the femoropopliteal arterial section.

With the median LEACSs of the total lower extremity and each arterial section as cutoffs, the patients were further allocated into high and low CS groups (Table 3). As shown by analysis of the total lower extremity and each arterial section, the LEACSs of patients aged $>60$ years were higher in the total lower extremity and all arterial sections. Furthermore, the LEACSs in the total lower extremity and aortoiliac arterial section were higher in patients with hypertension, whereas those in the femoropopliteal and infrapopliteal arterial sections were higher in patients with diabetes. Interestingly, there were more male patients in the high CS group of the infrapopliteal arterial section.

As shown by univariate linear regression analysis, the total LEACS was associated with age $>60$ years, diabetes, and hypertension. Multivariate analysis indicated that only age $>60$ years was an independent factor influencing the total LEACS. To determine the risk factors for different arterial sections, univariate and multivariate linear regression analyses were performed for each section of the lower extremity. After adjustment for age, the results showed that hypertension was an independent influencing factor for the aortoiliac LEACS, whereas diabetes was an independent influencing factor for the femoropopliteal and infrapopliteal LEACSs. In addition, male sex was an independent risk factor for the infrapopliteal LEACS (Tables 4 and 5).

\section{Discussion}

In this study, the total LEACS was associated with the total LEASI, indicating that the extent of arterial stenosis 


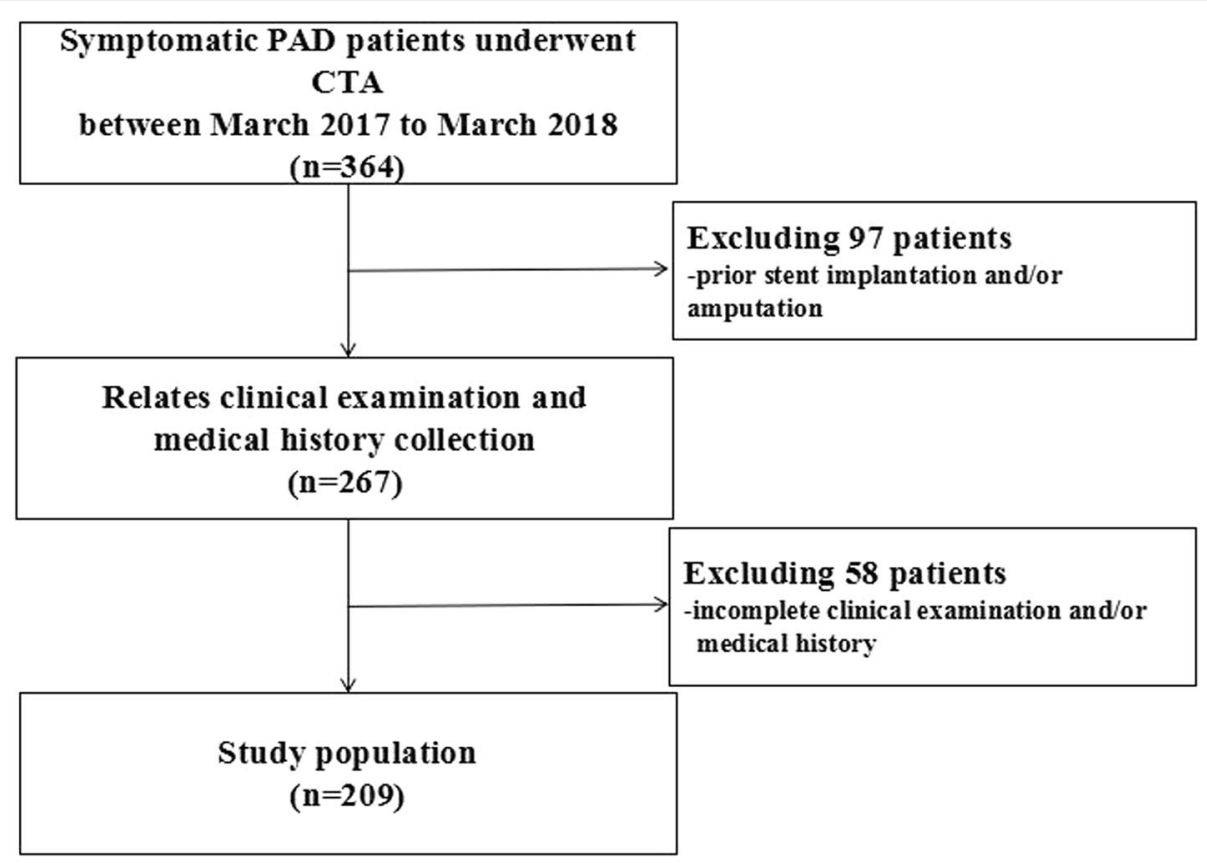

Fig. 1 Patient selection flow chart

could be better predicted by the degree of calcification. This finding is supported by several similar observations $[23,27]$. The internal diameter of blood vessels in the normal human body can be maintained through a compensatory increase in arterial perimeter, but Sigrist et al.

Table 1 Basic characteristics of the patients

\begin{tabular}{ll}
\hline Characteristics & PAD patients \\
\hline Number of patients ( $\mathrm{n})$ & 209 \\
Age (year) & $66.3 \pm 10.2$ \\
Age $>60$ years [n (\%)] & $149(71.3)$ \\
Smoking [n (\%)] & $148(70.8)$ \\
Drinking [n (\%)] & $92(44.0)$ \\
Male [n (\%)] & $164(78.5)$ \\
Diabetes [n (\%)] & $112(55.3)$ \\
Hyperlipidemia [n (\%)] & $93(53.6)$ \\
Hypertension [n (\%)] & $133(63.6)$ \\
CKD [n (\%)] & $49(23.4)$ \\
Total LEACS & $3.7 \pm 0.8$ \\
Aortoiliac LEACS & $3.5 \pm 0.7$ \\
Femoropopliteal LEACS & $3.0 \pm 1.2$ \\
Infrapopliteal LEACS & $2.5 \pm 1.6$ \\
Total LEASI & $12.6 \pm 7.2$ \\
AortoiliacLEASI & $1.1 \pm 1.4$ \\
Femoropopliteal LEASI & $4.9 \pm 3.2$ \\
Infrapopliteal LEASI & $6.7 \pm 5.0$ \\
\hline
\end{tabular}

Note: CKD chronic kidney disease, LEACS lower extremity arterial calcification score, LEASI lower extremity arterial stenosis index
[28] found that decreased arterial compliance is associated with femoral arterial calcification in dialysis patients, which might eventually lead to vascular stenosis. Similar results have been obtained in several studies $[29,30]$. Furthermore, we found that the LEACS was associated with the LEASI at the aortoiliac, femoropopliteal, and infrapopliteal arterial sections but showed a relatively weak correlation in the aortoiliac artery. This may be due to the different anatomical structures and hemodynamics of the lower extremity arteries, meaning that the influence of the calcification of different sections on the degree of arterial stenosis might differ. Because the diameter of the iliac artery is larger, it is less affected by the calcification of the vascular wall. In contrast, the distal femoropopliteal artery and infrapopliteal artery gradually become thinner, resulting in a greater influence of calcification.

Cardiovascular disease risk factors partially mediate the association between incident mortality and calcification in the different vascular beds [17]. The presence of calcification in the carotid or coronary arteries is indicative of a higher atherosclerotic burden and is associated with lower extremity arterial calcification in patients with PAD $[17,26]$. It would thus be valuable to describe the extent of the carotid or coronary arteral calcification. However, because only a few of the patients included in this study underwent carotid or coronary CT examination at the same time, only the lower extremity artery was involved in this study. The LEACS increased with age in patients with PAD in this study. Diabetes is associated with increased arterial calcification [31]. 

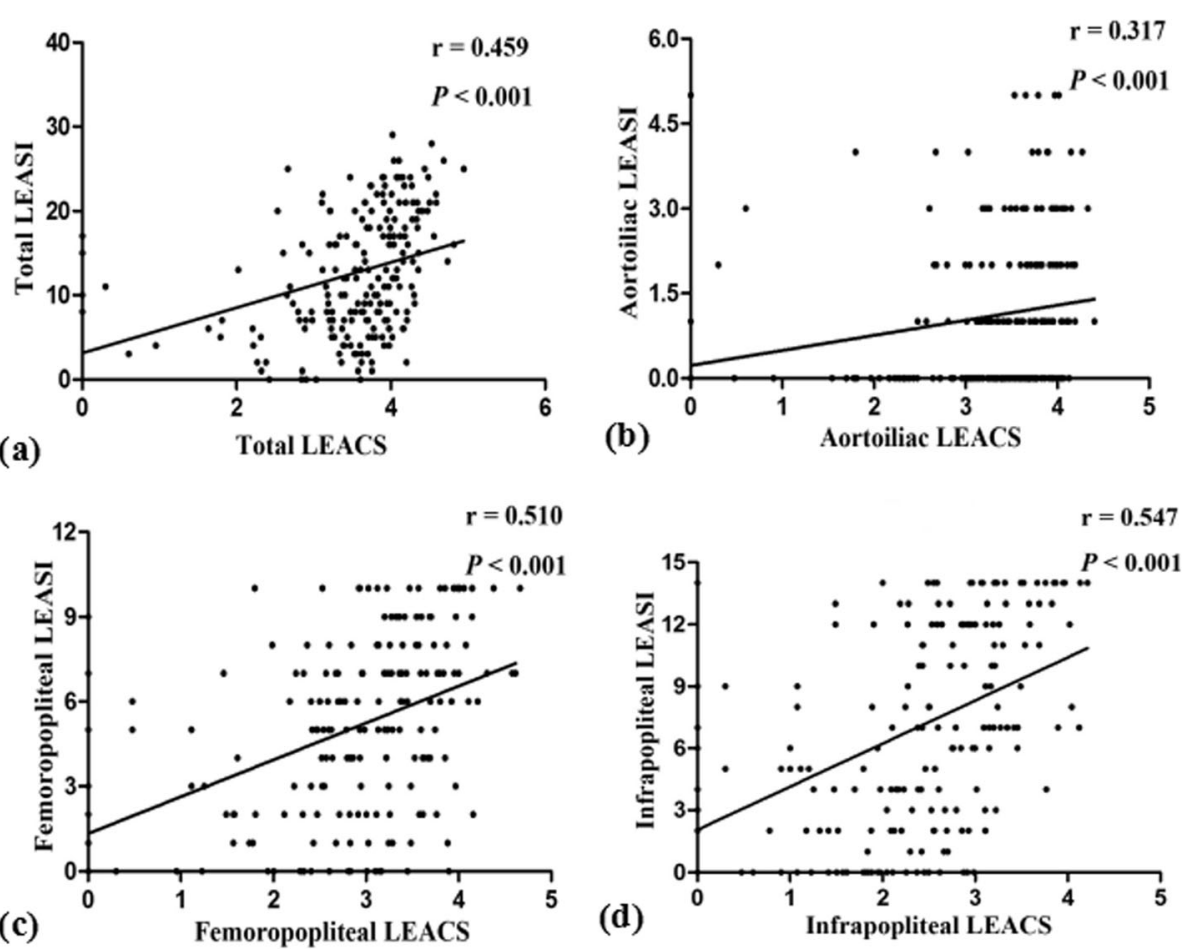

Fig. 2 Correlation between the LEACS and the LEASI. a Scatter diagram showing a positive correlation between the total LEACS and the total LEASI $(r=0.459, P<0.001)$. b Scatter diagram showing a positive correlation between the aortoiliac LEACS and the aortoiliac LEASI $(r=0.317, P<$ 0.001). c Scatter diagram showing a positive correlation between the femoropopliteal LEACS and the femoropopliteal LEASI $(r=0.510, P<0.001)$. d Scatter diagram showing a positive correlation between the infrapopliteal LEACS and the infrapopliteal LEASI $(r=0.547, P<0.001)$. LEACS, lower extremity arterial calcification score; LEASI, lower extremity arterial stenosis index

Hyperlipidemia is also a common risk factor for arterial calcification, especially in the coronary artery. In addition, some studies demonstrated that lipid-lowering agents could delay the calcification process [32]. Vascular calcification is more common and severe and develops earlier in patients with CKD or on dialysis [33]. However, the total LEACS was not significantly correlated with diabetes, CKD, or hypertension in this study, possibly because the sample size was insufficient or there was a sampling error.

The LEACS was also analyzed at each arterial section of the lower extremity in our study. In the high CS groups of the femoropopliteal and infrapopliteal arterial

Table 2 Correlation analysis of the LEACS among various sections

\begin{tabular}{lll}
\hline & $r$ & $P$ \\
\hline Total LEACS vs. Aortoiliac LEACS & 0.890 & $<0.001$ \\
Total LEACS vs. Femoropopliteal LEACS & 0.893 & $<0.001$ \\
Total LEACS vs. Infrapopliteal LEACS & 0.796 & $<0.001$ \\
Total LEACS vs. Left LEACS & 0.976 & $<0.001$ \\
Total LEACS vs. Right LEACS & 0.974 & $<0.001$ \\
Left LEACS vs. Right LEACS & 0.931 & $<0.001$ \\
\hline
\end{tabular}

Note: LEACS lower extremity artery calcification score sections, the proportions of patients $>60$ years old and with diabetes were higher; meanwhile, in the high CS group of the aortoiliac arterial section, the proportion of patients $>60$ years old and with hypertension was higher. As shown by multivariate linear regression analysis, after adjustment for age, the independent influencing factors for each arterial section of the lower extremity were different: hypertension and diabetes were independent risk factors for the aortoiliac LEACS and the femoropopliteal and infrapopliteal LEACSs, respectively, suggesting that the mechanisms for calcification at different arterial sections of the lower extremity might not be entirely the same and that the distribution of the LEACS is influenced by different risk factors. Previous work showed that compensatory arterial dilation in diabetic patients was restrained to exacerbate distal arteriosclerosis during the progression process of PAD [34]. Therefore, distal arterial calcification of the lower extremity is more serious in diabetic patients. In PAD patients with hypertension, the hemodynamic change plays a dominant role in the occurrence of lesions, so the proximal arteries of the lower extremity are more greatly influenced and may thus be more severely calcified, which correlates with our findings. In addition, male sex was also an independent risk factor for a higher infrapopliteal LEACS in this 
Table 3 Comparison of baseline data between the high and low CS groups of the whole lower extremity and various arterial sections

\begin{tabular}{|c|c|c|c|c|c|c|c|c|c|c|c|c|}
\hline & \multicolumn{3}{|c|}{ Total lower extremity } & \multicolumn{3}{|c|}{ Aortoiliac artery } & \multicolumn{3}{|c|}{ Femoropopliteal artery } & \multicolumn{3}{|c|}{ Infrapopliteal artery } \\
\hline & $\begin{array}{l}\text { High CS } \\
(n=104)\end{array}$ & $\begin{array}{l}\text { Low CS } \\
(n=105)\end{array}$ & $p$ & $\begin{array}{l}\text { High CS } \\
(n=104)\end{array}$ & $\begin{array}{l}\text { Low CS } \\
(n=105)\end{array}$ & $p$ & $\begin{array}{l}\text { High CS } \\
(n=104)\end{array}$ & $\begin{array}{l}\text { Low CS } \\
(n=105)\end{array}$ & $p$ & $\begin{array}{l}\text { High CS } \\
(n=104)\end{array}$ & $\begin{array}{l}\text { Low CS } \\
(n=105)\end{array}$ & $p$ \\
\hline Age $>60$ years & $84(80.8)$ & $65(61.9)$ & 0.003 & $84(80.8)$ & $65(61.9)$ & 0.003 & $85(81.7)$ & $64(61.0)$ & 0.001 & $83(79.8)$ & $66(62.9)$ & 0.00 \\
\hline Male & $85(81.7)$ & $79(75.2)$ & 0.254 & $86(82.7)$ & $78(74.3)$ & 0.139 & $82(78.8)$ & $82(78.1)$ & 0.837 & $90(86.5)$ & $74(70.5)$ & 0.01 \\
\hline Smoking & $76(73.1)$ & $72(68.6)$ & 0.474 & $80(76.9)$ & $68(64.8)$ & 0.053 & $70(67.3)$ & $78(74.3)$ & 0.267 & $73(70.2)$ & $75(71.4)$ & 0.84 \\
\hline Drinking & $49(47.1)$ & $43(41.0)$ & 0.370 & $49(47.1)$ & $43(41.0)$ & 0.370 & $48(46.2)$ & $44(41.9)$ & 0.536 & $49(47.1)$ & $43(41.0)$ & 0.37 \\
\hline Diabetes & $61(58.7)$ & $51(48.6)$ & 0.144 & $56(53.8)$ & $56(53.3)$ & 0.941 & $63(60.6)$ & $49(46.7)$ & 0.044 & $67(64.4)$ & $45(42.9)$ & 0.002 \\
\hline Hypertension & $75(72.1)$ & $58(55.2)$ & 0.011 & $74(71.2)$ & $59(56.2)$ & 0.025 & $71(68.3)$ & $62(59.0)$ & 0.166 & $67(64.4)$ & $66(62.9)$ & 0.81 \\
\hline Hyperlipidemia & $46(44.2)$ & $47(44.8)$ & 0.938 & $45(43.3)$ & $48(45.7)$ & 0.722 & $43(41.3)$ & $50(47.6)$ & 0.362 & $44(42.3)$ & 49 (46.7) & 0.52 \\
\hline CKD & $25(24.0)$ & $24(22.9)$ & 0.840 & $23(22.1)$ & $26(24.8)$ & 0.652 & $28(26.9)$ & $21(20.0)$ & 0.238 & $25(24.0)$ & $24(22.9)$ & 0.84 \\
\hline
\end{tabular}

Note: CS calcification score, CKD chronic kidney disease

study, and the proportion of male sex was higher in the high CS group of the infrapopliteal arterial section, which might be related to estrogen. As an important physiological hormone for female development, estrogen can increase bone density by inhibiting the activity of osteoclasts and thus also plays an important role in the metabolism of bone tissue. Previous studies have shown that serum estradiol levels are negatively correlated with coronary arterial calcification and that arterial calcification can be reduced by estrogen therapy $[35,36]$. Other work revealed that patient prognosis was influenced by arterial calcification in different sections of the lower extremity [37, 38]. Therefore, clinicians might preliminarily judge the distribution and prognosis of LEAC by identifying patients' relevant risk factors. In addition, the calcification of different sections might reflect the existence of different disease states. Furthermore, although arterial calcification is related to the degree of arterial stenosis, there is no clinically targeted drug that inhibits calcification, and its progress can thus only be indirectly slowed down through risk factor control. Our results suggest that, in future clinical practice, we will be able to control the related risk factors on a patient-specific basis according to the characteristics of calcification distribution in the lower extremity arteries.

Although the LEACS can effectively predict prognosis $[5,26]$, it is very time-consuming to measure the LEACS in the whole lower extremity artery in the clinical setting. In this study, the LEACS at each arterial section of the lower extremity and in both lower extremities were highly correlated with the total LEACS, especially in the femoropopliteal arterial sections. This suggests that it might be feasible to use the LEACS of a single arterial section, such as the femoropopliteal arterial section, to represent the total LEACS. Sook et al. [4] found that increased calcification of the tibial artery was associated with an increase in the failure rate of angioplasty and the incidence of nonscheduled amputation. Another study also suggested that calcification of the suprapatellar artery was associated with severe limb ischemia in patients on hemodialysis $[4,10]$. However, these associations should be further investigated in a large-scale study, given that the studies were single-center studies with small sample size and number of adverse events.

There are some limitations to our work. This study was a retrospective, small-sample, single-center study,

Table 4 Univariate linear regression analysis of the LEACS in the total lower extremity artery and at various arterial sections

\begin{tabular}{|c|c|c|c|c|c|c|c|c|}
\hline \multirow[t]{2}{*}{ Factors } & \multicolumn{2}{|c|}{ Total lower extremity artery } & \multicolumn{2}{|l|}{ Aortoiliac artery } & \multicolumn{2}{|l|}{ Femoropopliteal artery } & \multicolumn{2}{|l|}{ Infrapopliteal artery } \\
\hline & PRC (95\%Cl) & $P$ & PRC (95\%Cl) & $P$ & PRC $(95 \% \mathrm{Cl})$ & $P$ & PRC $(95 \% \mathrm{Cl})$ & $P$ \\
\hline Age $>60$ years & $0.744(0.496-0.992)$ & $<0.001$ & $0.650(0.397-0.903)$ & $<0.001$ & $0.988(0.650-1.325)$ & $<0.001$ & $0.840(0.503-1.177)$ & $<0.001$ \\
\hline Male & $0.50(-0.043-0.543)$ & 0.094 & $0.184(-0.110-0.478)$ & 0.219 & $0.199(-0.201-0.598)$ & 0.328 & $0.504(0.119-0.890)$ & 0.011 \\
\hline Diabetes & $0.263(0.023-0.504)$ & 0.032 & $0.098(-0.145-0.341)$ & 0.429 & $0.564(0.244-0.885)$ & 0.001 & $0.504(0.189-0.820)$ & 0.002 \\
\hline Smoking & $0.100(-0.167-0.366)$ & 0.461 & $0.238(-0.027-0.503)$ & 0.078 & $-0.004(-0.366-0.358)$ & 0.983 & $-0.051(0.405-0.303)$ & 0.778 \\
\hline Drinking & $0.090(-0.154-0.334)$ & 0.469 & $0.099(-0.145-0.343)$ & 0.425 & $0.152(-0.179-0.482)$ & 0.367 & $0.312(-0.009-0.634)$ & 0.057 \\
\hline CKD & $0.099(-0.187-0.385)$ & 0.496 & $-0.116(-0.402-0.170)$ & 0.424 & $0.310(-0.076-0.696)$ & 0.114 & $0.369(-0.008-0.746)$ & 0.055 \\
\hline Hypertension & $0.297(0.048-0.546)$ & 0.019 & $0.320(0.072-0.568)$ & 0.012 & $0.269(-0.071-0.609)$ & 0.120 & $0.170(-0.164-0.504)$ & 0.317 \\
\hline Hyperlipidemia & $0.0 .93(-0.151-0.337)$ & 0.452 & $0.121(-0.122-0.365)$ & 0.327 & $0.170(-0.161-0.500)$ & 0.313 & $-0.043(-0.367-0.281)$ & 0.795 \\
\hline
\end{tabular}


Table 5 Multivariate linear regression analysis of the LEACS in the total lower extremity artery and at various arterial sections

\begin{tabular}{|c|c|c|c|c|c|c|c|c|}
\hline \multirow[t]{2}{*}{ Factors } & \multicolumn{2}{|c|}{ Total lower extremity artery } & \multicolumn{2}{|l|}{ Aortoiliac artery } & \multicolumn{2}{|c|}{ Femoropopliteal artery } & \multicolumn{2}{|l|}{ Infrapopliteal artery } \\
\hline & PRC $(95 \% \mathrm{Cl})$ & $P$ & PRC (95\%Cl) & P & PRC $(95 \% \mathrm{Cl})$ & $P$ & PRC $(95 \% \mathrm{Cl})$ & P \\
\hline Age $>60$ years & $0.703(0.456-0.951)$ & $<0.001$ & $0.616(0.362-0.869)$ & $<0.001$ & $0.950(0.620-1.281)$ & $<0.001$ & $0.889(0.560-1.219)$ & $<0.001$ \\
\hline Male & - & - & - & - & - & - & $0.556(0.188-0.924)$ & 0.003 \\
\hline Diabetes & $0.184(-0.043-0.411)$ & 0.112 & - & - & $0.507(0.207-0.806)$ & 0.001 & $0.450(0.155-0.745)$ & 0.003 \\
\hline Hypertension & $0.194(-0.040-0.429)$ & 0.104 & $0.242(0.006-0.477)$ & 0.045 & - & - & - & - \\
\hline
\end{tabular}

which might lead to bias. Another important limitation was that, due to the retrospective design, we were unable to obtain detailed information on whether patients were treated systematically for combined disease. This might be important because some studies have shown that metformin and statins can inhibit calcification [32], which might affect the results. Nonetheless, this is the first study to analyze calcification in different lower limb sections, which could help to better explain the pathophysiology of arterial calcification. Further work with a larger sample size is nonetheless needed.

\section{Conclusions}

The LEACS is associated with the extent of arterial stenosis in all arterial sections, although the association was relatively weak in the aortoiliac artery. Traditional cardiovascular risk factors have varying effects on calcification among the different arterial sections of the lower extremity artery. Our results suggest that we should control the corresponding risk factors according to the distribution characteristics of LEAC to delay the calcification process.

\section{Abbreviations}

PAD: Peripheral arterial disease; LEACS: Lower extremity arterial calcification score; LEASI: Lower extremity arterial stenosis index; CT: Computed tomography; CTA: Computed tomography angiography; CKD: Chronic kidney disease; LEAC: Lower extremity arterial calcification; CS: Calcification score; PRC: Partial regression coefficient; Cl: Confidence interval

\section{Acknowledgements}

Not applicable.

\section{Authors' contributions}

Study conception and design was performed by HKY, ZHC, and ZYL. Data collection was performed by HKY and ZHC. Statistical analysis and the interpretation of the data were performed by HKY and ZHC. HKY wrote the first draft of the paper; ZHC, ZYL revised the article. This manuscript was read and approved by all credited authors.

\section{Funding}

The Young Scholar Support Program of China Medical University (QGZ2018053) supported the design of the study and data collection. The Doctoral Start-up Foundation of Liaoning Province (2019-BS-286) supported data analysis and manuscript writing.

\section{Availability of data and materials}

The datasets used and/or analyzed during the current study are available from the corresponding author on reasonable request.
Ethics approval and consent to participate

The study complied with the Declaration of Helsinki and was approved by the Ethics Committee of Shengjing hospital of China Medical University. The present study retrospectively analyzed data abstracted from the hospital information system of Shengjing Hospital of China Medical University without interventions or disruptions to patients' lives, and no direct human participants were involved, thus informed consent was not required and waived by the Ethics Committee.

\section{Consent for publication}

Not applicable.

\section{Competing interests}

The authors declare that they have no competing interests.

Received: 27 November 2019 Accepted: 7 July 2020

Published online: 11 July 2020

\section{References}

1. Fowkes FG, Rudan D, Rudan I, Aboyans V, Denenberg JO, McDermott MM, et al. Comparison of global estimates of prevalence and risk factors for peripheral artery disease in 2000 and 2010: a systematic review and analysis. Lancet. 2013;382(9901):1329-40.

2. GBD 2013 Mortality and Causes of Death Collaborators. Global, regional, and national age-sex specific all-cause and cause-specific mortality for 240 causes of death, 1990-2013: a systematic analysis for the global burden of disease study 2013. Lancet. 2015;385(9963):117-71.

3. Olin JW, White CJ, Armstrong EJ, Kadian-Dodov D, Hiatt WR. Peripheral artery disease: evolving role of exercise, medical therapy, and endovascular options. J Am Coll Cardiol. 2016;67(11):1338-57.

4. Kang IS, Lee W, Choi BW, Choi D, Hong MK. Jang Y1, Ko YG. Semiquantitative assessment of tibial artery calcification by computed tomography angiography and its ability to predict infrapopliteal angioplasty outcomes. J Vasc Surg. 2016;64(5):1335-43.

5. Huang $\mathrm{CL}$, Wu IH, Wu YW, Hwang JJ, Wang SS, Chen WJ, et al. Association of lower extremity arterial calcification with amputation and mortality in patients with symptomatic peripheral artery disease. PLoS One. 2014;9(2): e90201.

6. Block GA, Raggi P, Bellasi A, Kooienga L, Spiegel DM. Mortality effect of coronary calcification and phosphate binder choice in incident hemodialysis patients. Kidney Int. 2007;71(5):438-41.

7. Bostrom K. Bone morphogenetic protein expression in human atherosclerotic lesions. J Clin Invest. 1993:91(4):1800-9.

8. Thompson B, Towler DA. Arterial calcification and bone physiology: role of the bone-vascular axis. Nat Rev Endocrinol. 2012;8(9):529-43.

9. Demer LL, Tintut $Y$. Inflammatory, metabolic, and genetic mechanisms of vascular calcification. Arterioscler Thromb Vasc Biol. 2014:34(4):715-23.

10. Chen NX, Moe SM. Vascular calcification: pathophysiology and risk factors. Curr Hypertens Rep. 2012;14(3):228-37.

11. Pohle K, Mäffert R, Ropers D, Moshage W, Stilianakis N, Daniel WG, et al. Progression of aortic valve calcification: association with coronary atherosclerosis and cardiovascular risk factors. Circulation. 2001;104(16): 1927-32.

12. Bild DE, Detrano R, Peterson D, Guerci A, Liu K, Shahar E, et al. Ethnic differences in coronary calcification: the multi-ethnic study of atherosclerosis (MESA). Circulation. 2005;111(10):1313-20.

13. Chistiakov DA, Myasoedova VA, Melnichenko AA, Grechko AV, Orekhov AN. Calcifying matrix vesicles and atherosclerosis. Biomed Res Int. 2017;2017: 7463590 . 
14. Kelly-Arnold A, Maldonado N, Laudier D, Aikawa E, Cardoso L, Weinbaum S. Revised microcalcification hypothesis for fibrous cap rupture in human coronary arteries. Proc Natl Acad Sci U S A. 2013;110(26):10741-6.

15. Lanzer $P$, Boehm M, Sorribas $V$, Thiriet $M$, Janzen J, Zeller $T$, et al. Medial vascular calcification revisited: review and perspectives. Eur Heart J. 2014; 35(23):1515-25.

16. Amann K. Media calcification and intima calcification are distinct entities in chronic kidney disease. Clin J Am Soc Nephrol. 2008;3(6):1599-605.

17. Allison MA, Hsi S, Wassel CL, Morgan C, Ix JH, Wright CM, et al. Calcified atherosclerosis in different vascular beds and the risk of mortality. Arterioscler Thromb Vasc Biol. 2012;32(1):140-6.

18. Guerci AD, Spadaro LA, Popma JJ, Goodman KJ, Brundage BH, Budoff M, et al. Relation of coronary calcium score by electron beam computed tomography to arteriographic findings in asymptomatic and symptomatic adults. Am J Cardiol. 1997:79(2):128-33.

19. Park MS, Kwon S, Lee MJ, Kim KH, Jeon P, Park YJ, et al. Identification of high risk carotid artery stenosis: a multimodal vascular and perfusion imaging study. Front Neurol. 2019;10:765.

20. Otsuka F, Sakakura K, Yahagi K, Joner M, Virmani R. Has our understanding of calcification in human coronary atherosclerosis progressed? Arterioscler Thromb Vasc Biol. 2014;34(4):724-36.

21. Ohtake T, Oka M, Ikee R, Mochida Y, Ishioka K, Moriya H, et al. Kobayashi S impact of lower limbs' arterial calcification on the prevalence and severity of PAD in patients on hemodialysis. J Vasc Surg. 2011;53(3):676-83.

22. Chowdhury MM, Makris GC, Tarkin JM, Joshi FR, Hayes PD, Rudd JHF, et al. Lower limb arterial calcification (LLAC) scores in patients with symptomatic peripheral arterial disease are associated with increased cardiac mortality and morbidity. PLoS One. 2017;12(9):e0182952

23. Zettervall SL, Marshall AP, Fleser P, Guzman RJ. Association of arterial calcification with chronic limb ischemia in patients with peripheral artery disease. J Vasc Surg. 2018;67(2):507-13.

24. Itoga NK, Kim T, Sailer AM, Fleischmann D, Mell MW. Lower extremity computed tomography angiography can help predict technical success of endovascular revascularization in the superficial femoral and popliteal artery. J Vasc Surg. 2017:66(3):835-843.e1.

25. Chang Z, Yan H, Zhen Y, Zheng J, Liu Z. Lower limb arterial calcification and acute thrombosis risk in patients with peripheral artery disease. Ann Vasc Surg. 2019;63:227. https://doi.org/10.1016/j.avsg.2019.06.043.

26. Shin HS, Jung Park M, Nyeo Jeon K, Min Cho J, Soo Bae K, Seob Choi D, et al. Lower extremity arterial calcification as a predictor of coronary atherosclerosis in patients with peripheral arterial disease. Iran J Radiol. 2016;13(2):e33179.

27. Mackey RH, Venkitachalam L, Sutton-Tyrrell K. Calcifications, arterial stiffness and atherosclerosis. Adv Cardiol. 2007:44:234-44.

28. Sigrist MK, Mclntyre CW. Vascular calcification is associated with impaired microcirculatory function in chronic haemodialysis patients. Nephron Clin Pract. 2008:108(2):c121-6.

29. Yoshimura T, Suzuki E, Ito I, Sakaguchi M, Uzu T, Nishio Y, et al. Impaired peripheral circulation in lower-leg arteries caused by higher arterial stiffness and greater vascular resistance associates with nephropathy in type 2 diabetic patients with normal ankle-brachial indices. Diabetes Res Clin Pract. 2008;80(3):416-23.

30. Ahimastos AA, Dart AM, Lawler A, Blombery PA, Kingwell BA. Reduced arterial stiffness may contribute to angiotensin-converting enzyme inhibitor induced improvements in walking time in peripheral arterial disease patients. J Hypertens. 2008;26(5):1037-42.

31. Mary A, Hartemann A, Liabeuf S, Aubert CE, Kemel S, Salem JE, et al. Association between metformin use and below-the-knee arterial calcification score in type 2 diabetic patients. Cardiovasc Diabetol. 2017; 16(1):24

32. Asami M, Yamaji K, Aoki J, Tanimoto S, Watanabe M, Horiuchi Y, et al. Association of dyslipidemia and sex with coronary artery calcium assessed by coronary computed tomography angiography. Int Heart J. 2017;58(5): 695-703.

33. Rostand SG, Drueke TB. Parathyroid hormone, vitamin D, and cardiovascular disease in chronic renal failure. Kidney Int. 1999:56(2):383-92.

34. Lehto S, Niskanen L, Suhonen M, Rönnemaa T, Laakso M. Medial artery calcification. A neglected harbinger of cardiovascular complications in noninsulin-dependent diabetes mellitus. Arterioscler Thromb Vasc Biol. 1996 16(8):978-83.
35. Jeon GH, Kim SH, Yun SC, Chae HD, Kim CH, Kang BM. Association between serum estradiol level and coronary artery calcification in postmenopausal women. Menopause. 2010;17(5):902-7.

36. Manson JE, Allison MA, Rossouw JE, Carr JJ, Langer RD, Hsia J, et al. Estrogen therapy and coronary-artery calcification. N Engl J Med. 2007; 356(25):2591-602

37. McDermott MM, Kramer CM, Tian L, Carr J, Guralnik JM, Polonsky T, et al. Plaque composition in the proximal superficial femoral artery and peripheral artery disease events. JACC Cardiovasc Imaging. 2017;10(9):1003-12.

38. Guzman RJ, Brinkley DM, Schumacher PM, Donahue RM, Beavers H, Qin X. Tibial artery calcification as a marker of amputation risk in patients with PAD. J Am Coll Cardiol. 2008;51(20):1967-74

\section{Publisher's Note}

Springer Nature remains neutral with regard to jurisdictional claims in published maps and institutional affiliations.
Ready to submit your research? Choose BMC and benefit from:

- fast, convenient online submission

- thorough peer review by experienced researchers in your field

- rapid publication on acceptance

- support for research data, including large and complex data types

- gold Open Access which fosters wider collaboration and increased citations

- maximum visibility for your research: over $100 \mathrm{M}$ website views per year

At $\mathrm{BMC}$, research is always in progress.

Learn more biomedcentral.com/submissions 